\title{
Accuracy and Precision of Compartmental Model Parameters Obtained from Directly Estimated Dynamic SPECT Time-Activity Curves
}

\author{
Bryan W. Reutter, Member, IEEE, Grant T. Gullberg, Senior Member, IEEE, \\ and Ronald H. Huesman, Fellow, IEEE
}

\begin{abstract}
Quantitative kinetic analysis of dynamic cardiac single photon emission computed tomography (SPECT) data has the potential to provide better contrast between healthy and diseased tissue, compared to static images. However, imaging a rapidly changing radiopharmaceutical distribution with the use of a moving gantry yields inconsistent projection data that can generate artifacts in a time sequence of conventional image reconstructions. The artifacts can lead to biases in kinetic parameters estimated from the image sequence. This source of bias can be eliminated by estimating B-spline models for time-activity curves directly from the projections. In this study, we perform Monte Carlo simulations to determine how the polynomial order and initial time sampling of the splines affect the accuracy and precision of compartmental model parameters obtained from directly estimated time-activity curves. The Mathematical Cardiac Torso (MCAT) phantom is used to simulate a realistic $15 \mathrm{~min}$ dynamic ${ }^{99 \mathrm{~m}} \mathrm{Tc}$-teboroxime patient study in which 10 million total events are detected. For a large volume of normal myocardium $(250 \mathrm{cc})$, the relative bias of the uptake and washout parameter sample means does not exceed $0.22 \%$ when using cubic or quadratic splines that provide rapid initial sampling. The coefficient of variation is about $1 \%$. For small $(8.4 \mathrm{cc})$ myocardial defects that exhibit reduced uptake and accelerated washout, the relative bias and coefficient of variation increase to maximum values of about $16 \%$ and $49 \%$, respectively. These levels of accuracy and precision allow the defects to be discriminated from the normal myocardium.
\end{abstract}

Index Terms-dynamic single photon emission computed tomography (SPECT), fully four-dimensional (4-D) reconstruction, kinetic parameter estimation

\section{INTRODUCTION}

Q UANTITATIVE kinetic analysis of dynamic cardiac single photon emission computed tomography (SPECT) data has the potential to provide better contrast between healthy and diseased tissue, compared to static images [1]. However, imaging a rapidly changing radiopharmaceutical distribution with the use of a moving gantry yields inconsistent projection

Manuscript received December 2, 2002. This work was supported by the National Heart, Lung, and Blood Institute of the U.S. Department of Health and Human Services under grants R01-HL50663 and P01-HL25840 and by the Director, Office of Science, Office of Biological and Environmental Research, Medical Sciences Division of the U.S. Department of Energy under contract DE-AC03-76SF00098. This work was developed in part with the use of resources at the U.S. Department of Energy National Energy Research Scientific Computing (NERSC) Center.

The authors are with the Department of Nuclear Medicine and Functional Imaging, Lawrence Berkeley National Laboratory, University of California, Berkeley, CA 94720 USA (e-mail: bwreutter@lbl.gov). data that can generate artifacts in a time sequence of conventional image reconstructions. The artifacts can lead to biases in kinetic parameters estimated from time-activity curves obtained by overlaying volumes of interest on the image sequence. This source of bias can be eliminated by faithfully modeling the time variation of the activity distribution throughout the projected field of view and estimating temporal model parameters directly from the projection data [2]-[10].

Previously, we used B-splines to model the time courses of activity within segmented volumes and developed fast methods to estimate spline model coefficients and their statistical uncertainties directly from dynamic SPECT projection data [6], [10]. In the present work, we perform Monte Carlo simulations of a realistic dynamic cardiac ${ }^{99 \mathrm{~m}} \mathrm{Tc}$-teboroxime patient study to determine how the polynomial order and initial time sampling of the splines affect the accuracy and precision of compartmental model parameters obtained from directly estimated timeactivity curves.

\section{Methods}

\section{A. Direct Temporal B-Spline Model Estimation}

Smooth time-activity curves for segmented volumes encompassing the projected field of view can be estimated directly from dynamic SPECT projection data as follows. This method can be applied to projection data acquired with the use of any collimator or orbit geometry, provided that the data yield a preliminary image reconstruction that can be used to segment the activity distribution throughout the projected field of view.

The spline model for the time-activity curve for segmented volume $m$ is

$$
A^{m}(t)=\sum_{n=1}^{N} a_{m n} V^{n}(t)
$$

where $a_{m n}$ are model coefficients, $V^{n}(t)$ are B-spline basis functions [11], and $N$ is the number of basis functions. Splines with smaller support typically are used to model rapidly changing portions of curves, while splines with larger support are used to model slow changes (e.g., [12]).

The detected count rate at time $t$ along ray $i$ is modeled as

$$
P_{i}(t)=\sum_{m=1}^{M} U_{i}^{m}(t) A^{m}(t)=\sum_{m=1}^{M} \sum_{n=1}^{N} a_{m n} U_{i}^{m}(t) V^{n}(t),
$$


where $U_{i}^{m}(t)$ is the spatial projection, along ray $i$, of the indicator function for volume $m$ and $M$ is the number of volumes encompassing the projected field of view.

The model for the projection data is obtained by integrating (2) over $L$ contiguous time intervals that span the data acquisition from time $t_{0}=0$ to time $t_{L}=T$ :

$$
p_{i l}=\sum_{m=1}^{M} \sum_{n=1}^{N} a_{m n} \int_{t_{l-1}}^{t_{l}} U_{i}^{m}(\tau) V^{n}(\tau) d \tau .
$$

If the time intervals are short enough so that each segmented volume projection function $U_{i}^{m}(t)$ is approximated well by a piecewise constant function with amplitude $u_{i l}^{m}$ during time interval $\left[t_{l-1}, t_{l}\right]$, then the model for the projection data can be simplified:

$$
p_{i l}=\sum_{m=1}^{M} \sum_{n=1}^{N} a_{m n} u_{i l}^{m} v_{l}^{n}
$$

where $v_{l}^{n}$ are the integrals $\int_{t_{l-1}}^{t_{l}} V^{n}(\tau) d \tau$ of the temporal Bspline basis functions.

The temporal spline model coefficients $a_{m n}$ are estimated by minimizing the sum of squared differences between the measured and modeled projections:

$$
\chi^{2}=\sum_{i=1}^{I} \sum_{l=1}^{L}\left(p_{i l}^{*}-p_{i l}\right)^{2},
$$

where $p_{i l}^{*}$ are the measured projections and $I$ is the number of projection rays acquired simultaneously by the detector(s). For a periodic (e.g., multi-rotation circular) orbit, the spline model coefficients $a_{m n}$, their covariance matrix, and the global precision of time-activity curve models can be estimated using fast methods that take advantage of periodicity in the $u_{i l}^{m}$ factors [6], [10].

\section{B. Nonlinear Compartmental Modeling}

Directly estimated spline models yield smooth time-activity curves that can have a variety of shapes. In many cases the relationship between the time-activity curves for the blood pool and a tissue volume of interest is described accurately by a compartmental model. The compartmental model parameters have physiological meaning and can provide a quantitative measure of tissue perfusion [1]. It has been hypothesized that compartmental analysis may also be useful for assessing tissue viability [4].

For the one-compartment kinetic model (Fig. 1), the relationship between the blood input function, $B(t)$, and the activity in the tissue in volume $m, Q^{m}(t)$, is modeled to be

$$
\frac{d Q^{m}(t)}{d t}=k_{21}^{m} B(t)-k_{12}^{m} Q^{m}(t)
$$

where $k_{21}^{m}$ is the uptake rate parameter and $k_{12}^{m}$ is the washout rate parameter. For initial conditions of zero, the tissue activity

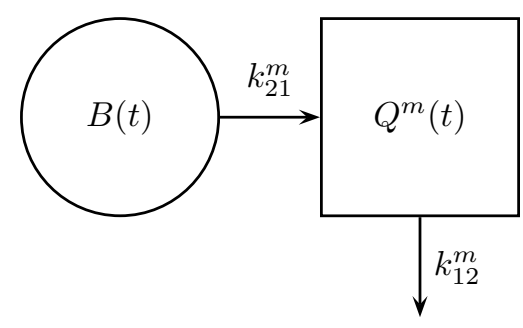

Fig. 1. Compartmental model for ${ }^{99 \mathrm{~m}} \mathrm{Tc}$-teboroxime in the myocardium.

is the convolution of the blood input function with a single decaying exponential:

$$
Q^{m}(t)=k_{21}^{m} \int_{0}^{t} B(\tau) e^{-k_{12}^{m}(t-\tau)} d \tau=k_{21}^{m} C^{m}(t) .
$$

Total activity in volume $m$ is given by $k_{21}^{m} C^{m}(t)+f_{v}^{m} B(t)$, where $f_{v}^{m}$ is the fraction of vasculature in the volume.

The compartmental model parameters $k_{21}^{m}, k_{12}^{m}$, and $f_{v}^{m}$ are estimated by minimizing the sum of squared differences between the spline and compartmental models:

$$
\chi_{m}^{2}=\sum_{l=1}^{L}\left\{\sum_{n=1}^{N} \hat{a}_{m n} v_{l}^{n}-\int_{t_{l-1}}^{t_{l}}\left[k_{21}^{m} \hat{C}^{m}(\tau)+f_{v}^{m} \hat{B}(\tau)\right] d \tau\right\}^{2}
$$

where $\hat{a}_{m n}$ are values for spline model coefficients that minimize (5), $\sum_{n=1}^{N} \hat{a}_{m n} v_{l}^{n}$ is the integral of the temporal spline model for total activity in volume $m$ during time interval $\left[t_{l-1}, t_{l}\right], \hat{C}^{m}(\tau)$ is the convolution $\int_{0}^{\tau} \hat{B}\left(\tau^{\prime}\right) e^{-k_{12}^{m}\left(\tau-\tau^{\prime}\right)} d \tau^{\prime}$, and $\hat{B}(\tau)$ is derived from the temporal spline model for activity in the blood pool.

\section{COMPuter Simulations}

We performed Monte Carlo simulations of a realistic dynamic cardiac ${ }^{99 \mathrm{~m}} \mathrm{Tc}$-teboroxime patient study acquired on a single-detector SPECT system. The goal was to determine how the polynomial order and initial time sampling of the temporal B-spline basis functions affect the accuracy and precision of compartmental model parameters obtained from directly estimated time-activity curves.

Simulated spatial distributions were obtained with the use of the Mathematical Cardiac Torso (MCAT) phantom [13]. The emission phantom (Fig. 2) was composed of 128 contiguous $1.75 \mathrm{~mm}$-thick slices and contained $M=6$ volumes of interest: the blood pool, three myocardial tissue volumes (normal myocardium, septal defect, and lateral defect), liver, and background tissue. Each volume was modeled to contain spatially uniform activity.

The simulated time-activity curves for the six emission volumes are shown in Fig. 3. The time-activity curves for the three myocardial volumes of interest and the liver were generated by using the blood pool curve as the input to onecompartment models that have kinetics corresponding to those of ${ }^{99 \mathrm{~m}} \mathrm{Tc}$-teboroxime [14]. 


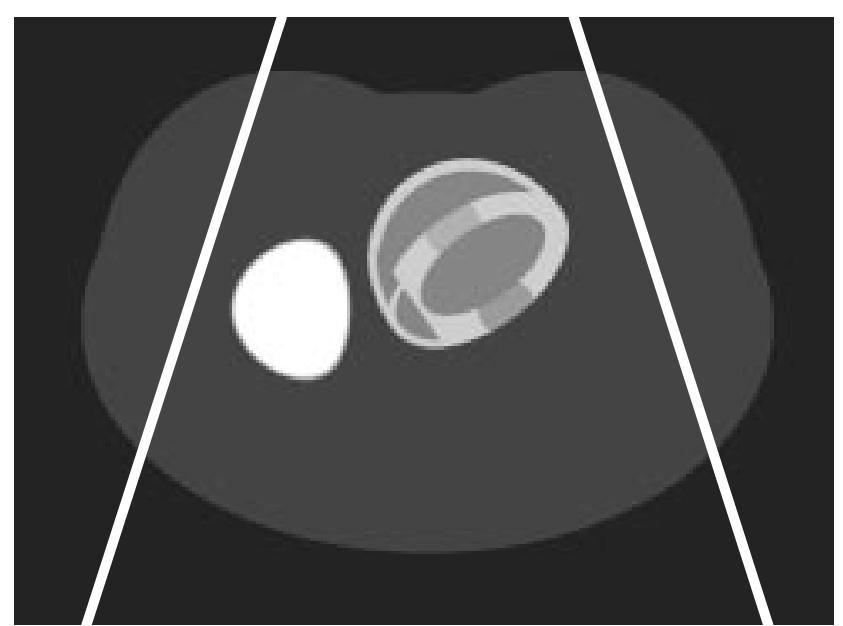

Fig. 2. Transverse cross section through MCAT emission phantom. White lines depict data truncation that results from use of cone beam collimators.

The simulated 15-min dynamic SPECT data acquisition consisted of one $360^{\circ}$ circular rotation per minute and 120 projection angles per rotation. Projection data were obtained simultaneously along $I=2048$ rays (64 transverse $\times 32$ axial) during each of $L=1800$ contiguous 0.5 -s time intervals, to yield a total of about 3.7 million projection measurements. The projection bins were $7 \mathrm{~mm} \times 7 \mathrm{~mm}$ at the detector, which was $30 \mathrm{~cm}$ from the center of the field of view. The detector was offset $1 \mathrm{~cm}$ from cone beam collimators that had a hole diameter of $2 \mathrm{~mm}$ and a length of $4 \mathrm{~mm}$. The focal length of the collimators was $70 \mathrm{~cm}$, which resulted in truncation of the data (Fig. 2). Projections were attenuated with the use of the corresponding MCAT attenuation phantom. Attenuation and geometric point response were modeled with the use of a raydriven projector with line length weighting [15]. Scatter was not modeled. The amplitude of the blood input function was adjusted so that about 10 million total events were detected.

For each of 24 sets of temporal B-spline basis functions, time-activity curve models were estimated directly from 1000 realizations of data having Poisson noise. Each set of basis functions consisted of $N=16$ splines that span 15 time segments having geometrically increasing length (e.g., Fig. 4). Piecewise cubic, quadratic, linear, or constant B-splines were used with initial time segment lengths of $2.5,5,10,20,40$, or $60 \mathrm{~s}$. The shorter initial time segment lengths provided a higher density of temporal spline basis functions at the beginning of the simulated acquisition, when the activity concentrations were changing most rapidly (Fig. 3). The $60 \mathrm{~s}$ initial time segment length provided basis functions spaced uniformly in time. The cubic, quadratic, and linear B-splines allowed modeling of curves that were continuous through their second, first, and zeroth derivative, respectively.

Given a temporal B-spline basis set and a noisy realization of projection data, we calculated the $96 a_{m n}$ coefficients, their covariance matrix, and the global precision of time-activity

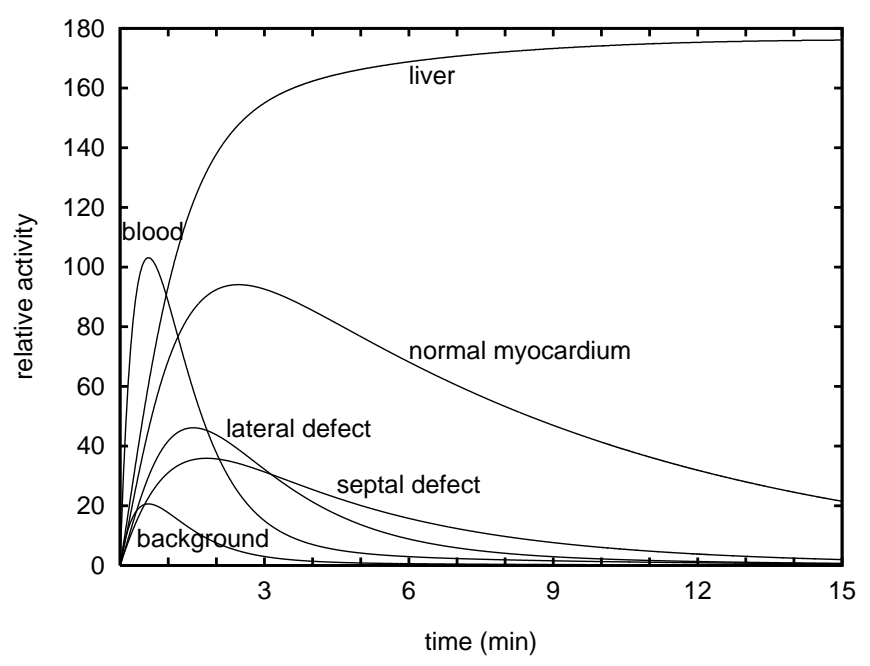

Fig. 3. Simulated ${ }^{99 \mathrm{~m}}$ Tc-teboroxime time-activity curves.

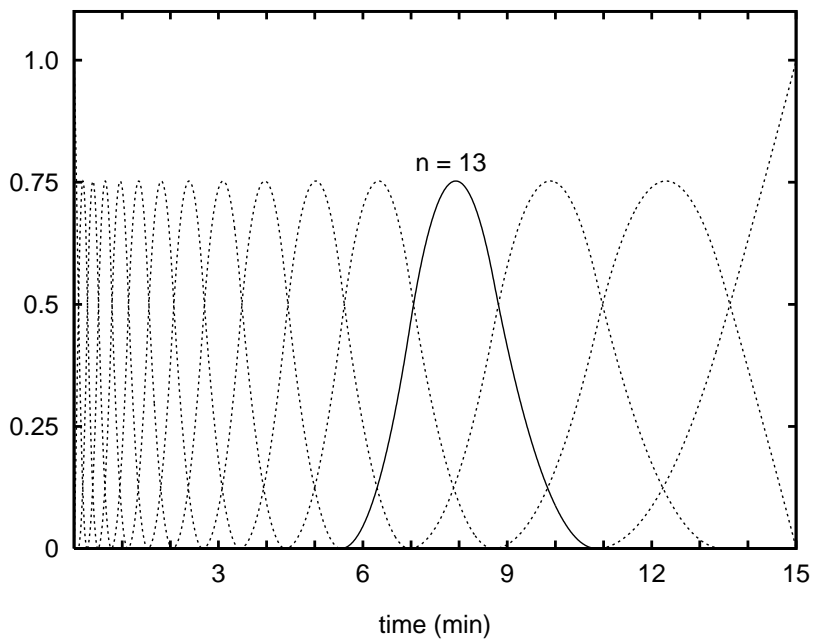

Fig. 4. Sixteen piecewise quadratic B-spline temporal basis functions that have an initial time segment length of $10 \mathrm{~s}$. The thirteenth spline is shown as a solid curve.

curve models in $34 \mathrm{~s}$ using a 194-MHz R10000-based SGI workstation and the fast methods presented in [6], [10]. We estimated compartmental model parameters from the spline time-activity curve models in $18 \mathrm{~s}$ using the program RFIT [16].

The computer simulation results are presented in Figs. 5 and 6. For each compartmental model parameter, relative bias was calculated as the magnitude of the difference between the sample mean and the simulated value, normalized by the simulated value. The coefficient of variation was calculated by normalizing the sample standard deviation by the simulated value. The use of cubic, quadratic, or linear splines and an initial time sampling of $20 \mathrm{~s}$ or less reduced compartmental model parameter bias substantially without unduly increasing the coefficient of variation.

For the large volume of normal myocardium $(250 \mathrm{cc})$, the relative bias of the sample mean of the uptake and washout 

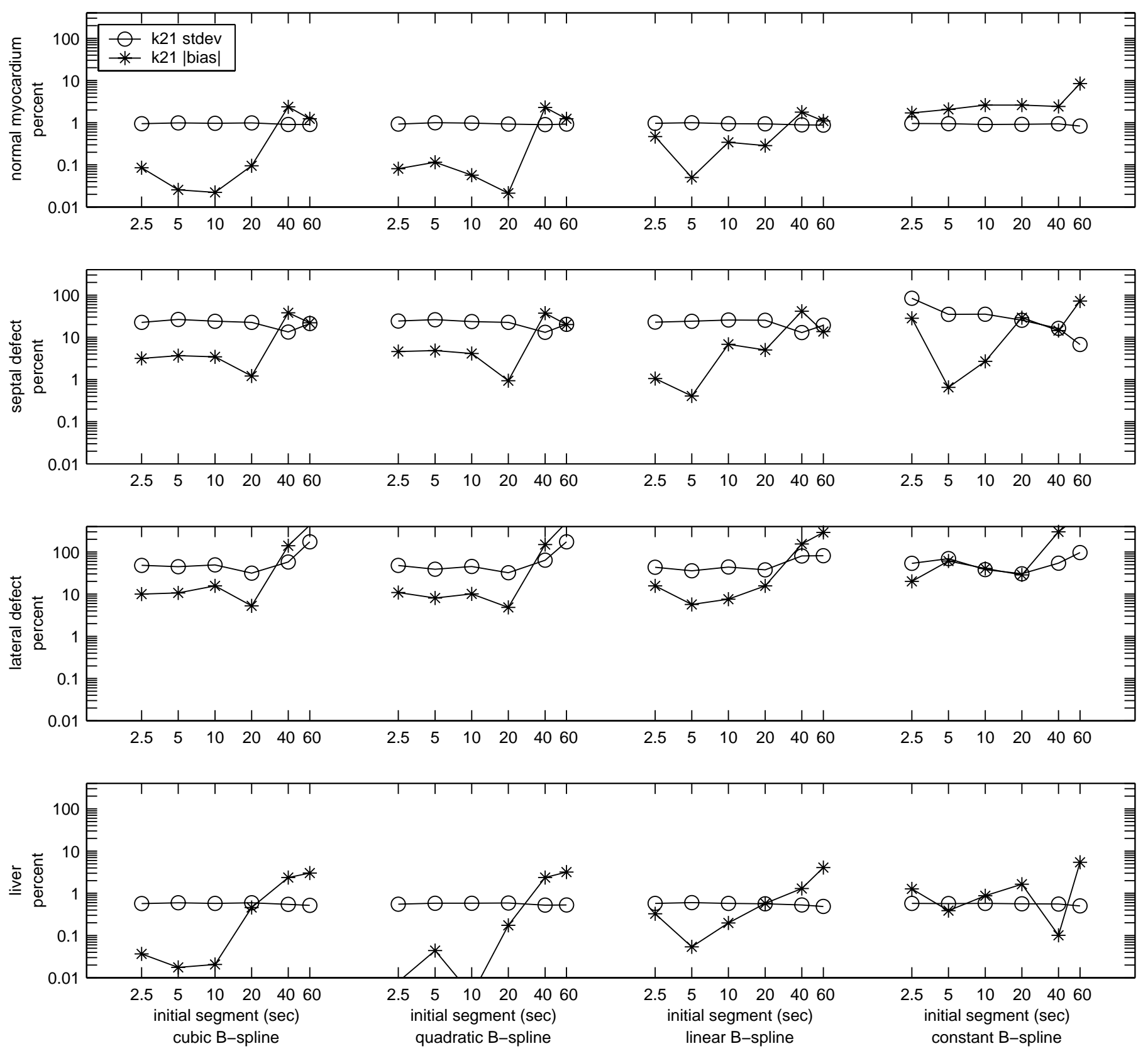

Fig. 5. Effects of temporal spline modeling on the tissue uptake parameter, $k_{21}^{m}$. The simulated values for normal myocardium, septal defect, lateral defect, and liver were $0.7,0.3,0.5$, and $0.9 \mathrm{~min}^{-1}$, respectively. The "*” symbols denote relative bias values observed for 1000 realizations of noisy projections. The "o" symbols denote the observed coefficients of variation.

parameters did not exceed $0.22 \%$, for cubic or quadratic splines and an initial time sampling of $20 \mathrm{~s}$ or less. The coefficient of variation of the parameters was about $1 \%$.

For the small $(8.4 \mathrm{cc})$ defects that exhibit reduced uptake and accelerated washout, the relative bias and coefficient of variation increased to maximum values of about $16 \%$ and $49 \%$, respectively, for cubic or quadratic splines and an initial time sampling of $20 \mathrm{~s}$ or less. The increases were due to the relatively small size of the defects and the relatively small amplitude and temporal support of their time-activity curves. Nonetheless, these levels of accuracy and precision allowed the defects to be discriminated from the normal myocardium.

For the large volume of liver $(1100 \mathrm{cc})$ that exhibits the largest uptake, the relative bias and coefficient of variation of the uptake parameter did not exceed $0.46 \%$ and $0.60 \%$, respectively, for cubic or quadratic splines and an initial time sampling of $20 \mathrm{~s}$ or less. Because the 500-min time constant for washout from the liver is extremely long relative to the 15 -min duration of the simulated study (Fig. 3), the relative bias and coefficient of variation of the washout rate parameter increased 

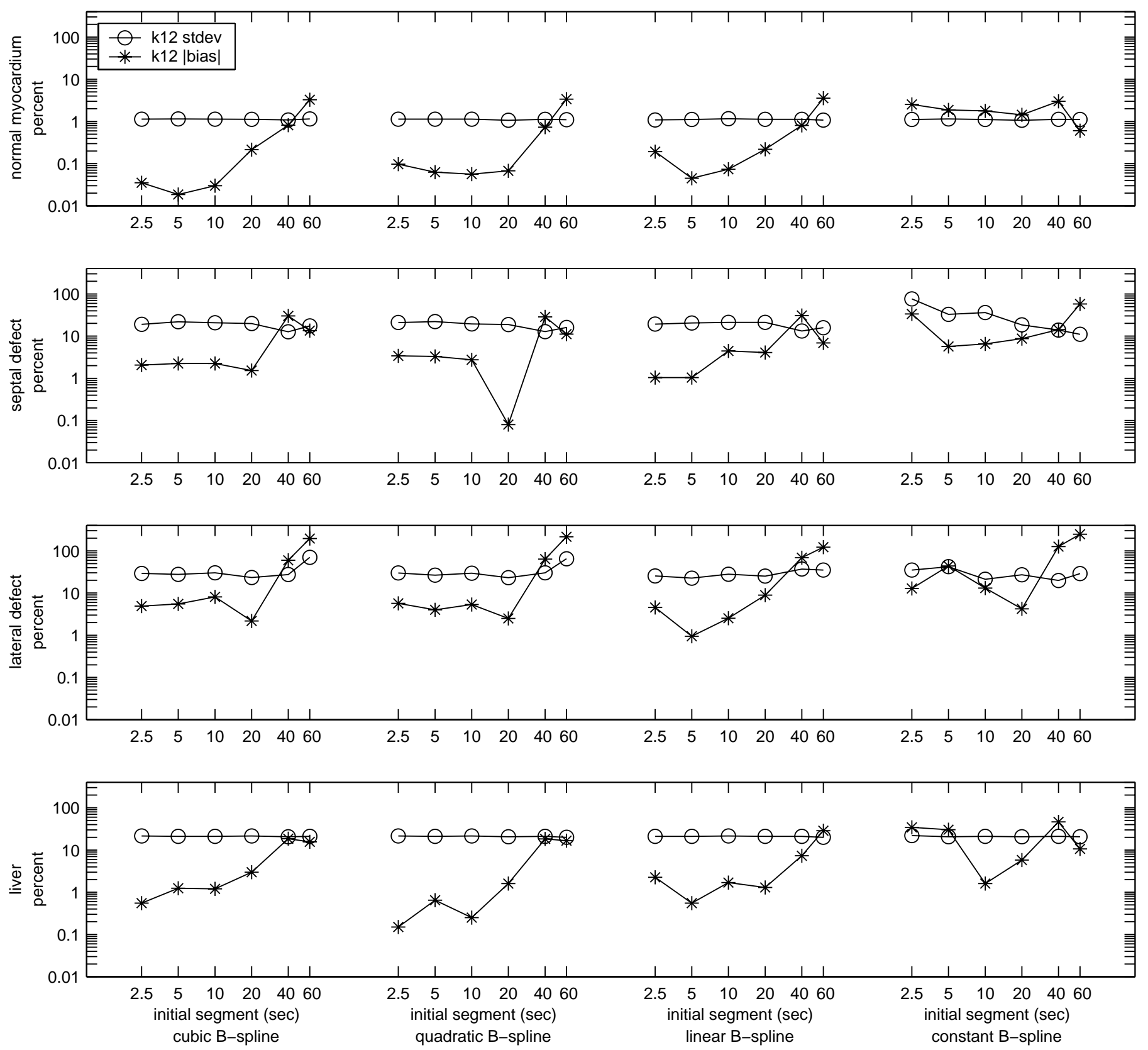

Fig. 6. Effects of temporal spline modeling on the tissue washout parameter, $k_{12}^{m}$. The simulated values for normal myocardium, septal defect, lateral defect, and liver were $0.15,0.3,0.6$, and $0.002 \mathrm{~min}^{-1}$, respectively. The "*” symbols denote relative bias values observed for 1000 realizations of noisy projections. The "o" symbols denote the observed coefficients of variation.

to maximum values of about $3 \%$ and $22 \%$, respectively, for cubic or quadratic splines and an initial time sampling of $20 \mathrm{~s}$ or less. The absolute bias and standard deviation were relatively small, however.

\section{Discussion}

The computer simulation results presented in Section III suggest that accurate and precise estimates of compartmental model parameters for relatively large tissue volumes can be obtained quickly from time-activity curves estimated directly from dynamic SPECT projection data. Piecewise cubic or quadratic B-spline basis functions can model typical time-activity curves accurately and provide desired temporal regularization.

As part of future work, parameter estimates can be refined by jointly estimating the blood time-activity spline curve and tissue compartmental models directly from projection data. Incorporation of the compartmental relationship between blood and tissue activities into the model for the projection data will provide more temporal regularization than is provided by splines alone. For example, the use of splines as described 
in Section II-A introduces 16 parameters for each volume of interest and imposes no functional form on the relationship between the blood and tissue time-activity curves. By comparison, the use of one-compartment models introduces only three parameters for each tissue volume and constrains the tissue activity to be a convolution of the blood activity. This additional temporal regularization in the model for the projection data may improve the accuracy and precision of compartmental models for relatively small tissue volumes such as the myocardial defects simulated in Section III.

\section{ACKNOWLEDGMENT}

The authors thank Dr. Benjamin Tsui and his colleagues for making the MCAT phantom available.

This work was supported by the National Heart, Lung, and Blood Institute of the U.S. Department of Health and Human Services under grants R01-HL50663 and P01-HL25840 and by the Director, Office of Science, Office of Biological and Environmental Research, Medical Sciences Division of the U.S. Department of Energy under contract DE-AC03-76SF00098.

This work was developed in part with the use of resources at the U.S. Department of Energy National Energy Research Scientific Computing (NERSC) Center.

\section{REFERENCES}

[1] E. V. R. Di Bella, S. G. Ross, D. J. Kadrmas, H. S. Khare, P. E. Christian, S. McJames, and G. T. Gullberg, "Compartmental modeling of technetium-99m-labeled teboroxime with dynamic single-photon emission computed tomography: Comparison with static thallium-201 in a canine model," Invest. Radiol., vol. 36, no. 3, pp. 178-185, 2001.

[2] R. H. Huesman, B. W. Reutter, G. L. Zeng, and G. T. Gullberg, "Kinetic parameter estimation from SPECT cone-beam projection measurements," Phys. Med. Biol., vol. 43, no. 4, pp. 973-982, 1998.

[3] B. W. Reutter, G. T. Gullberg, and R. H. Huesman, "Kinetic parameter estimation from dynamic cardiac patient SPECT projection measurements," in 1998 IEEE Nuclear Science Symposium and Medical Imaging Conference Record, R. Sudharsanan, Ed., 1999, pp. 1953-1958.

[4] G. T. Gullberg, R. H. Huesman, S. G. Ross, E. V. R. Di Bella, G. L. Zeng, B. W. Reutter, P. E. Christian, and S. A. Foresti, "Dynamic cardiac single-photon emission computed tomography," in Nuclear Cardiology: State of the Art and Future Directions, B. L. Zaret and G. A. Beller, Eds. St. Louis: Mosby Inc., 1999, ch. 11, pp. 137-187.

[5] A. Sitek, E. V. R. Di Bella, and G. T. Gullberg, "Reconstruction from slow rotation dynamic SPECT using a factor model," in Information Processing in Medical Imaging: Proceedings of the 16th International Conference, A. Kuba, M. Šámal, and A. Todd-Pokropek, Eds., 1999, pp. 436-441.

[6] B. W. Reutter, G. T. Gullberg, and R. H. Huesman, "Direct leastsquares estimation of spatiotemporal distributions from dynamic SPECT projections using a spatial segmentation and temporal B-splines," IEEE Trans. Med. Imag., vol. 19, no. 5, pp. 434-450, 2000.

[7] T. Farncombe, A. Celler, C. Bever, D. Noll, J. Maeght, and R. Harrop, "The incorporation of organ uptake into dynamic spect (dSPECT) image reconstruction," IEEE Trans. Nucl. Sci., vol. 48, no. 1, pp. 3-9, 2001.

[8] D. J. Kadrmas and G. T. Gullberg, "4D maximum a posteriori reconstruction in dynamic SPECT using a compartmental model-based prior," Phys. Med. Biol., vol. 46, no. 5, pp. 1553-1574, 2001.

[9] J. S. Maltz, "Optimal time-activity basis selection for exponential spectral analysis: Application to the solution of large dynamic emission tomographic reconstruction problems," IEEE Trans. Nucl. Sci., vol. 48, no. 4, pp. 1452-1464, 2001.

[10] B. W. Reutter, R. H. Huesman, and G. T. Gullberg, "Effects of temporal modelling on the statistical uncertainty of spatiotemporal distributions estimated directly from dynamic SPECT projections," Phys. Med. Biol., vol. 47, no. 15, pp. 2673-2683, 2002.
[11] R. H. Bartels, J. C. Beatty, and B. A. Barsky, An Introduction to Splines for Use in Computer Graphics and Geometric Modeling. Los Altos, CA: Morgan Kaufmann Publishers, Inc., 1987.

[12] T. E. Nichols, J. Qi, and R. M. Leahy, "Continuous time dynamic PET imaging using list mode data," in Information Processing in Medical Imaging: Proceedings of the 16th International Conference, A. Kuba, M. Śámal, and A. Todd-Pokropek, Eds., 1999, pp. 98-111.

[13] B. M. W. Tsui, J. A. Terry, and G. T. Gullberg, "Evaluation of cardiac cone-beam single photon emission computed tomography using observer performance experiments and receiver operating characteristic analysis," Invest. Radiol., vol. 28, no. 12, pp. 1101-1112, 1993.

[14] R. K. Narra, T. Feld, and A. D. Nunn, "Absorbed radiation dose to humans from technetium-99m-teboroxime," J. Nucl. Med., vol. 33, no. 1, pp. 8893, 1992.

[15] G. L. Zeng, G. T. Gullberg, B. M. W. Tsui, and J. A. Terry, "Threedimensional iterative reconstruction algorithms with attenuation and geometric point response correction," IEEE Trans. Nucl. Sci., vol. 38, no. 2, pp. 693-702, 1991.

[16] R. H. Huesman, B. L. Knittel, B. M. Mazoyer, P. G. Coxson, E. M. Salmeron, G. J. Klein, B. W. Reutter, and T. F. Budinger, "Notes on RFIT: A program for fitting compartmental models to region-of-interest dynamic emission tomography data," Lawrence Berkeley National Laboratory, Tech. Rep. LBL-37621, 1995.

\section{DISCLAIMER}

This document was prepared as an account of work sponsored by the United States Government. While this document is believed to contain correct information, neither the United States Government nor any agency thereof, nor The Regents of the University of California, nor any of their employees, makes any warranty, express or implied, or assumes any legal responsibility for the accuracy, completeness, or usefulness of any information, apparatus, product, or process disclosed, or represents that its use would not infringe privately owned rights. Reference herein to any specific commercial product, process, or service by its trade name, trademark, manufacturer, or otherwise, does not necessarily constitute or imply its endorsement, recommendation, or favoring by the United States Government or any agency thereof, or The Regents of the University of California. The views and opinions of authors expressed herein do not necessarily state or reflect those of the United States Government or any agency thereof, or The Regents of the University of California.

Ernest Orlando Lawrence Berkeley National Laboratory is an equal opportunity employer. 